\title{
PET IMAGE RECONSTRUCTION USING PRIOR INFORMATION FROM CT OR MRI
}

\author{
Filip Šroubek*, Michal Šorel, Jiři Boldyš \\ UTIA, Academy of Sciences of CR \\ Pod Vodárenskou věží 4 \\ Prague 8, 182 08, Czech Republic
}

\author{
Jan Šroubek \\ Na Homolce Hospital, Dept. of Neurosurgery \\ Roentgenova 2 \\ Prague 5, 150 30, Czech Republic
}

\begin{abstract}
Functional properties of living tissues appear in PET, whereas structural information at significantly higher resolution and better image quality is provided by other modalities, such as CT or MRI. We illustrate how structural information of matched anatomic images can be used as priors in the total variation denoising and blind deconvolution of functional PET images. Experiments on phantom images and clinical data validate the proposed method.
\end{abstract}

Index Terms - PET, total variation, denoising, deconvolution, PET/CT, PET/MRI

\section{INTRODUCTION}

PET examination is a well established method in medicine, mostly for identifying lesions as being malignant or benign. Surgery for the lesion is almost always based on structural examination provided by MRI or CT. A problem for surgery appears if the existing pathological tissue is not identifiable by MRI/CT but can be seen by PET (as for example in some cases of epilepsy patient). As the PET scans are structurally not precise enough it is difficult for neurosurgeon to identify the brain lesion and there is a need for an invasive exploration of the brain, hence increasing the perioperative morbidity. Making the PET image more focused and less noisy would decrease the risk of surgery.

PET denoising methods based on the wavelet transform have been reported to increase accuracy and precision of PET images in a wide variety of contexts [1]. In order to provide further improvement of PET images, it was acknowledged that the structural information in MRI or CT can be used. Using structural information in image processing is not a new idea, e.g., denoising and deconvolution of images using predicted edge location was proposed recently in [2]. Boussion et al. [3] proposed denoising of PET images in the wavelet domain while using high frequency information from CT or MRI. This is achieved by replacing high-pass bands of PET, which are extremely noisy, with high-pass bands of the

*Financial support of this research was provided by Czech Ministry of Education under the project 1M0572 (Research Center DAR) and the Grant Agency of the Czech Republic under the project 102/08/1593. structural image, which contain details invisible in PET. The appropriate scaling between PET and MRI/CT wavelet coefficients at the finest resolution was obtained by assuming a global linear relationship between PET and MRI/CT wavelets at the lower resolution. Recently, Turkheimer et al. [4] proposed an improved method, which relaxes the assumption of a simple scalar relation between structural and functional information and postulated a stochastic model.

Apart from noise and low resolution, blurring is an additional significant factor that degrades PET images. Uncertainty caused by positron traveling distance, photon emission angle, scatter, and detector response can be model by a blurring function, i.e. convolution with a point spread function (PSF). If the shape of PSFs is unknown, we face a blind deconvolution problem. An attempt to tackle this complicated problem is given in [5], where an EM reconstruction technique incorporates the blurring function and estimates PSFs in the process. Recently, Siemens introduced the TrueX image processing technology, which reduces blurring introduced by fuzzy response of detectors further away from the center of the field of view.

In this paper, we formulate the PET reconstruction as a blind deconvolution and denoising problem constrained by structural information from MRI/CT. The novelty of the proposed method is in the way how the image prior is built, which consists of total variation (TV) seminorm of the MRI/CT data. The rest of the paper is organized as follows. Section 2 discusses the proposed method, Section 3 provides experiments, and Section 4 concludes the work.

\section{METHOD}

We assume that the relation between the unknown (sharp and clear) PET image $\mathbf{f}$ and the measured PET image $g$ is given in the vector-matrix form by

$$
\mathbf{g}=\mathbf{H f}+\mathbf{n},
$$

where $\mathbf{H}$ represents an unknown blurring matrix, and $\mathbf{n}$ represents additive noise. The blurring matrix $\mathbf{H}$ is fully determined by its PSF, which we denote as $\mathbf{h}$. All the images are 
assumed to be lexicographically ordered into column vectors with pixels addressed by a single index $i$.

The proposed denoising and blind deconvolution approach follows the Bayesian paradigm. The posterior distribution takes the form

$$
p(\mathbf{f}, \mathbf{h} \mid \mathbf{g}) \propto p(\mathbf{g} \mid \mathbf{f}, \mathbf{h}) p(\mathbf{f}) p(\mathbf{h})
$$

and based on its knowledge, the inference on the sharp image $\mathbf{f}$ and PSF $\mathbf{h}$ is done. Since the posterior distribution can not be calculated in closed form, the common strategy is to approximate it by a product $q(\mathbf{f}) q(\mathbf{h})$ and a variational approach is used to find expressions for the simplified distributions $q(\cdot)$. If $q(\cdot)$ 's are assumed to be delta functions, the variational approach becomes a maximum a posteriori (MAP) method. In our implementation we adopt the MAP approach. This leads to an iterative algorithm [6], which alternates between the maximization of (2) with respect to the image and the maximization with respect to PSF.

The first term in (2) emanates from our model. Assuming that the observation noise $\mathbf{n}$ in (1) is Gaussian with zero mean and variance $\alpha^{-1}$, we obtain

$$
p(\mathbf{g} \mid \mathbf{f}, \mathbf{h}) \propto \exp \left\{-\frac{1}{2} \alpha\|\mathbf{H} \mathbf{f}-\mathbf{g}\|^{2}\right\} .
$$

A difficult issue is the choice of prior distributions in (2). Typically, PSFs in PET are smooth and therefore we use simultaneous autoregression for the PSF prior distribution:

$$
p(\mathbf{h}) \propto \exp \left\{-\frac{1}{2} \beta\|\mathbf{L h}\|^{2}\right\},
$$

where the matrix $\mathbf{L}$ denotes the Laplacian operator and $\beta$ is the inverse variance of Gaussian distribution. This prior favors PSFs with small second derivatives, hence smooth functions.

However, the key idea of this paper is in the derivation of the PET image prior distribution $p(\mathbf{f})$. Total variation is a common choice for the image prior. It works as an anisotropic diffusion, which preserves edges. In the discrete case, the TV prior is defined as

$$
p(\mathbf{f}) \propto \exp \{-\gamma \mathrm{TV}(\mathbf{f})\}
$$

with

$$
\mathrm{TV}(\mathbf{f})=\sum_{i} \sqrt{\left(\Delta_{i}^{x} \mathbf{f}\right)^{2}+\left(\Delta_{i}^{y} \mathbf{f}\right)^{2}},
$$

where $\Delta_{i}^{x}$ and $\Delta_{i}^{y}$ represent first order differences in $x$ and $y$ direction, respectively, at the pixel position $i$. The derivative of TV is nonlinear and one must employ linearization techniques, such as half-quadratic algorithm [7], in order to work with this prior. For the purpose of our discussion it suffices to state that after linearization we obtain

$$
\mathrm{TV}(\mathbf{f})=\mathbf{f}^{\mathrm{T}} \mathcal{L} \mathbf{f}
$$

where $\mathcal{L}$ is a symmetric block diagonal matrix constructed from $\Delta_{i}^{x} \mathbf{f}$ and $\Delta_{i}^{y} \mathbf{f}$. Since $\mathcal{L}$ depends on $\mathbf{f}, \mathcal{L}$ is updated after every iteration of the standard half-quadratic algorithm. In smooth areas, $\mathcal{L}$ is isotropic and resembles the Laplacian operator, whereas on an edge, $\mathcal{L}$ becomes directional and this allows smoothing only along the edge but not across. Some object boundaries in the structural image coincide with boundaries barely visible in the functional image. Therefore it is legitimate to assume that the information about edges in the $\mathrm{MRI} / \mathrm{CT}$ image can be utilized in the PET prior. In order to derive the new PET prior, we thus use (3), but construct $\mathcal{L}$ from the MRI/CT image instead of the updated PET image in each iteration. We call it the STV (structural TV) prior and it gives higher probability to edges that coincide with MRI/CT edges. It is important to note that this does not mean that all MRI/CT edges will start to appear in the reconstructed PET image but only those, which are intrinsic to the PET image. This is a fundamental difference from the wavelet-based methods [3, 4], which replace noisy high-pass bands of PET with MRI/CT and insert thus fine details irrespective of their true existence in PET. Our method performs denoising and deconvolution of PET and uses MRI/CT only to control diffusion. An additional advantage of the STV method is that it is robust to misregistration between PET and MRI/CT as opposed to the wavelet-based methods. Since the proposed method also estimates PSFs, it can compensate for slight misalignment by automatically shifting the center of PSFs.

\section{RESULTS}

Performance of the proposed STV method was evaluated on two data sets: Jaszczak SPECT phantom [8] and abdominal section of a human body. Comparison was carried out with the state-of-the-art wavelet-based denoising method proposed in [4]. All the data were acquired by a hybrid PET/CT camera Siemens BIOGRAPH DUO LSO. PET images were first upsampled to the CT resolution and than processed by the wavelet-based and proposed reconstruction algorithm. Objective performace evaluation is difficult to carry out as we lack a sharp noise-free PET image. In the case of phantom images, we calculated PSNR and for the sharp PET image we used the CT modality correctly radiometrically transformed to match the intensities of the PET modality. However, visual (subjective) assessment still remains the most reliable evaluation method.

An example of one slice of the phantom volume acquired in both modalities (PET and CT) is given in Fig. 1. The phantom contains cold rods of different dimensions positioned into triangles oriented around the center. The 3D reconstruction method was OSEM (Ordered Subset Expectation Maximization) with 6 iterations and 16 subsets with no post-processing Gaussian filtering to avoid any additional blurring. Fig. 2 shows reconstruction by the wavelet-based method and by our STV method. Both methods give equal results in terms of de- 
noising. However, the proposed STV methods shows superior performance around edges with contours better localized. This fact also illustrates Fig. 3 by comparing line profiles of the reconstructed images. The position of the line, of which profiles are drawn, is shown in Fig. 1(a).

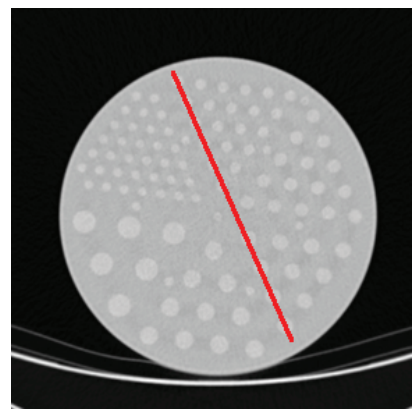

(a) CT

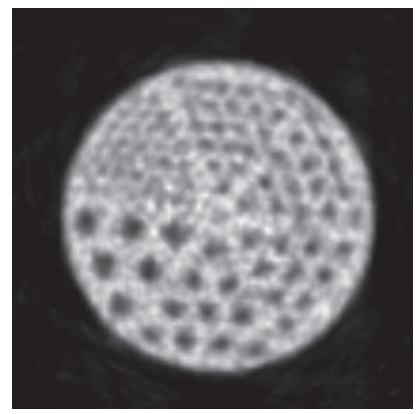

(b) PET
Fig. 1. Example of a phantom volume acquired by a combined PET/CT scanner: (a) CT $(512 \times 512 \times 159)$ image and $(\mathrm{b})$ corresponding PET $(256 \times 256 \times 47)$ image, PSNR = $24.91 \mathrm{~dB}$.

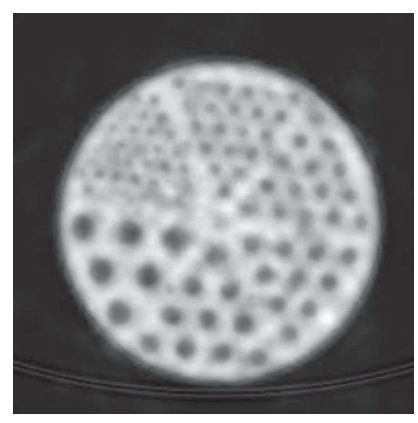

(a) WT-based reconstruction

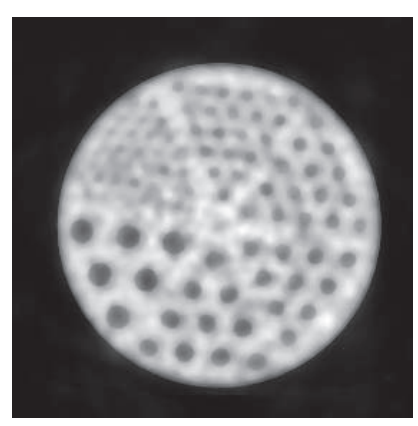

(b) STV-prior reconstruction
Fig. 2. Reconstruction of PET images using structural information from CT: (a) wavelet-based denoising approach proposed in [4] PSNR = 25.43dB, (b) our STV-prior denoising and deconvolution method PSNR $=25.39 \mathrm{~dB}$.

Combined PET/CT scanners provide both modalities in perfect spatial alignment. However, such scans are still rare and measurements must be done separately, not to mention that combined PET/MRI scanners do not exist. We must first apply registration methods to spatially align functional images with structural images, which is a complicated task. Often the registered modalities still contain some misalignment. Hence it is desirable if the denoising algorithm is robust to misregistration as is the case of the STV method. Fig. 4 illustrates the reconstruction when the CT image was artificially shifted by 5 pixels (approx. $5 \mathrm{~mm}$ ) in the $\mathrm{x}$ and $\mathrm{y}$ direction. The wavelet-based method removes noise but blurring remains, whereas our STV method provides the same sharp image as in the case with correct registration. Calcu-

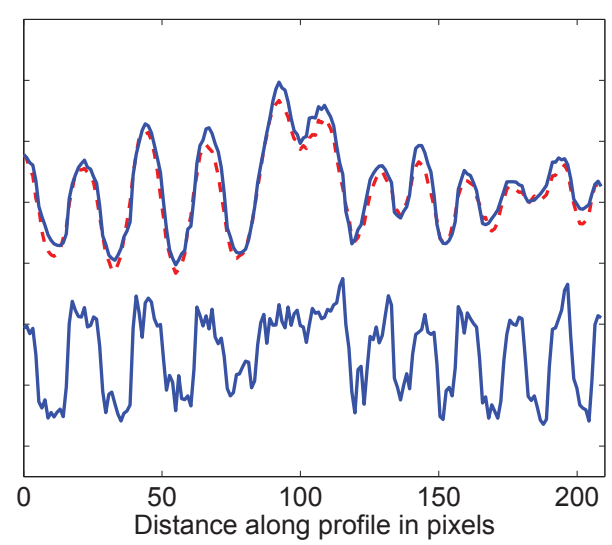

Fig. 3. Line profile at the location depicted by the solid line in Fig. 1 in: (bottom solid) CT image, (top dashed) WT-based reconstruction in Fig. 2a, (top solid) STV-prior reconstruction in Fig. $2 b$.

lated PSNRs confirm our visual assessment as PSNR of the wavelet-based method drops while PSNR of the STV method remains almost the same (see figure captions). Estimated PSFs in both experiments for properly aligned and shifted CT image, respectively, are in Fig. 5.

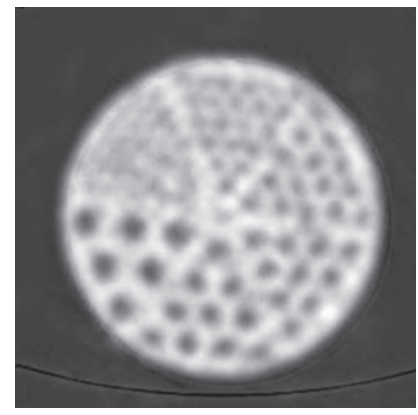

(a) WT-based reconstruction

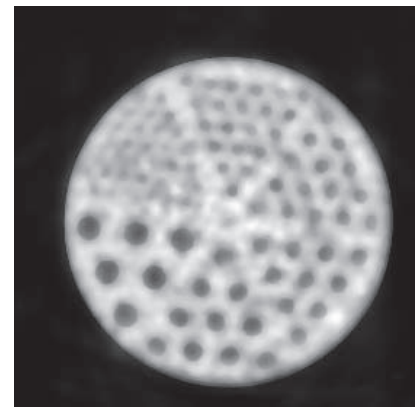

(b) STV-prior reconstruction
Fig. 4. Reconstruction of PET images using structural information from misaligned CT: (a) wavelet-based denoising approach proposed in [4] PSNR $=24.77 \mathrm{~dB}$, (b) our STV-prior denoising and deconvolution method PSNR $=25.17 \mathrm{~dB}$.

Finally, we applied the proposed STV method to a CT/PET pair of the abdominal section; see Fig. 6. In this case, the PET image was post-processed inside the CT/PET scanner by a Gaussian filter to reduce noise. For denoising methods it would be better to work with the original noisy PET image, but since this is a standard post-processing procedure used by physicians, we wanted to test if the method can be applied to readily available images. The wavelet-based denoising method was not able to improve the image quality and the results is not shown here. On the other hand, the output of the STV method shows several tumors in the liver 


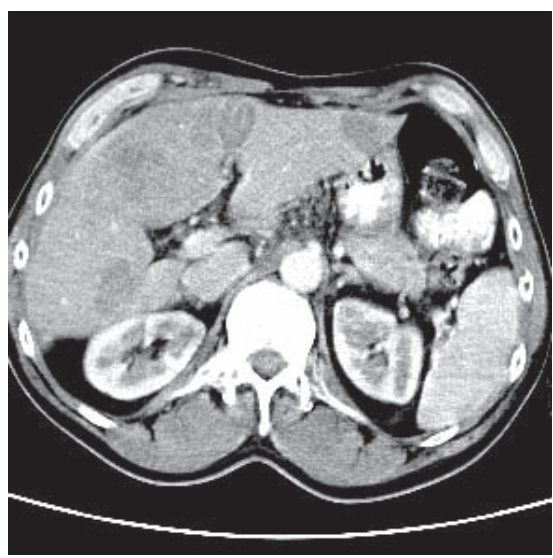

(a) CT

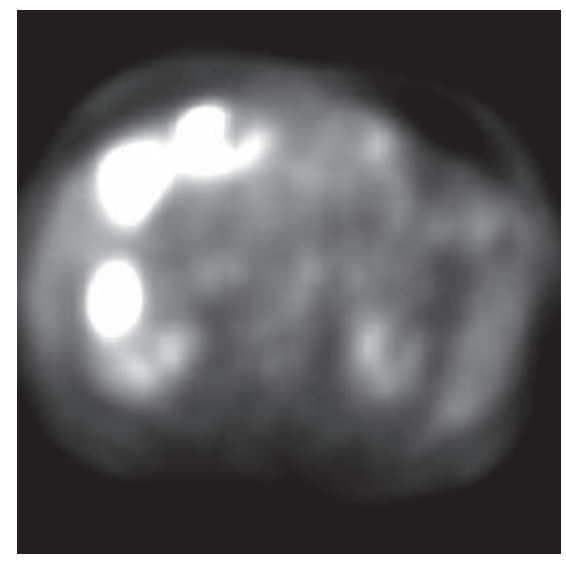

(b) PET

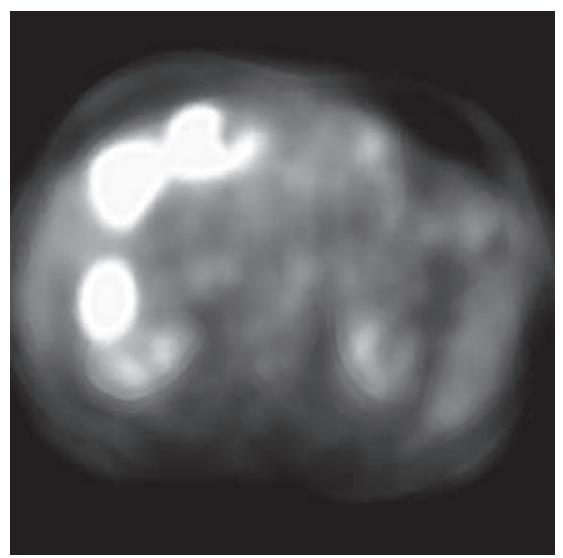

(c) STV-prior reconstruction

Fig. 6. Reconstruction of a PET image using structural information from CT: (a) CT image, (b) PET image, (c) STV-prior reconstruction.
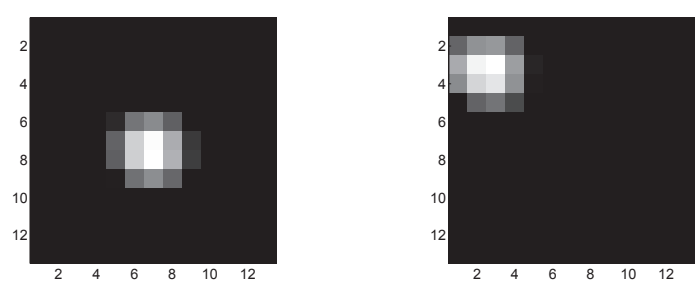

Fig. 5. PSF estimation in our STV-prior reconstruction method for (left) CT and PET accurately registered, (right) $\mathrm{CT}$ and PET with misalignment of $5 \mathrm{~mm}$ in both axes.

sharper and thus better localized.

\section{CONCLUSION}

We have proposed a denoising and blind deconvolution MAP method for PET images, which includes structural information from MRI/CT images as a modified TV prior distribution. The proposed method provides better focused PET images and it is robust to misalignment.

We plan to test the method further on other clinical data. The primary goal will be to evaluate whether the reconstruction algorithm works also on MRI/PET pairs and if the improved PET images can help to perform surgery.

\section{ACKNOWLEDGMENT}

The tomographic images were kindly provided by Dr. Otakar Belohlavek, Head of the Department of Nuclear Medicine, $\mathrm{Na}$ Homolce Hospital.

\section{REFERENCES}

[1] Nathaniel M Alpert, Anthonin Reilhac, Tat C Chio, and Ivan Selesnick, "Optimization of dynamic measurement of receptor kinetics by wavelet denoising.," Neuroimage, vol. 30, no. 2, pp. 444-451, Apr 2006.

[2] N. Joshi, R. Szeliski, and D. J. Kriegman, "Psf estimation using sharp edge prediction," in Proc. IEEE Conference on Computer Vision and Pattern Recognition CVPR 2008, 23-28 June 2008, pp. 1-8.

[3] N. Boussion, et al., "A multiresolution image based approach for correction of partial volume effects in emission tomography.," Phys Med Biol, vol. 51, no. 7, pp. 1857-1876, Apr 2006.

[4] F. E. Turkheimer, et al., "Pet image denoising using a synergistic multiresolution analysis of structural (MRI/CT) and functional datasets.," J Nucl Med, vol. 49, no. 4, pp. 657-666, Apr 2008.

[5] Heng Li, Feng Qiao, O. R. Mawlawi, Yibin Zheng, and R. X. Zhu, "Blind deblurring reconstruction technique with applications in pet imaging," in Proc. 4th IEEE International Symposium on Biomedical Imaging: From Nano to Macro ISBI 2007, 12-15 April 2007, pp. 173176.

[6] T.F. Chan and C.K. Wong, "Total variation blind deconvolution," IEEE Trans. Image Processing, vol. 7, no. 3, pp. 370-375, Mar. 1998.

[7] G. Aubert and P. Kornprobst, Mathematical Problems in Image Processing, Springer Verlag, New York, 2002.

[8] Biodex, "Jaszczak SPECT phantom," http://www.biodex.com/radio/phantoms/phantoms_750.htm. 